\title{
Mechanisms of critical current limitation in YBCO thin film structures
}

\author{
S. Benacka, V. Strbik, S. Chromik, R. Adam, M. Darula, and S. Gazi \\ Institute of Electrical Engineering, Slovak Academy of Sciences, 84239 Bratislava, Slovak Republic \\ E-mail:elekbena@savba.sk \\ Received March 10, 1998
}

\begin{abstract}
The limitation of critical current in high $T_{c}$ superconducting $\mathrm{YBCO}$ thin films has a varied nature according to the quality of $\mathrm{YBCO}$ films. Our results showed that in strips from granular films the weak links of superconductor-normal metal-superconductor and superconductor-constriction-superconductor types were responsible for critical current limitation. In the YBCO strips with improved (preferential) crystallographic orientation a flux-creep critical current limitation was found and in the highly oriented YBCO strips the Ginzburg-Landau depairing mechanism approximated the critical current limitation for temperature close to $T_{c}$.

PACS: 74.76.Bz, 74.72.Bk, 74.50.+r, 74.80.Fp
\end{abstract}

\section{Introduction}

The critical current (or critical current density) is one of the most important parameters of the transport electrical properties of high temperature superconducting thin film strips. Various critical current limiting mechanisms (CCLM) take part according to the thin film quality. The nature of CCLM can be recognized from the critical current vs. temperature dependence $\left(I_{c}(T)\right)$. The important contribution to these problems in low $T_{c}$ film strips have been made by the I. M. Dmitrenko group from Kharkov (see the excellent review paper [1] and papers cited therein). In this contribution we review some of our results concerning $I_{c}(T)$ studies on high $T_{c}$ superconducting thin films, strips and junctions, stimulated by Prof. I. M. Dmitrenko's results and personal contacts.

\section{Experimental, results and discussion}

The $\mathrm{YBa}_{2} \mathrm{Cu}_{3} \mathrm{O}_{7-x}$ (YBCO) thin films were deposited by dc magnetron sputtering or vacuum coevaporation method on $\mathrm{MgO}, \mathrm{SrTiO}_{3}$, or YSZ and $\mathrm{CeO}_{2}$ buffered $\mathrm{Al}_{2} \mathrm{O}_{3}$ single crystal substrates. The strips were patterned by wet or ion beam etching.

In Fig. 1 we summarize $I_{c}(T)$ dependencies in normalized units, where $I_{c}(T) / I_{c}(0) \equiv j_{c}(T) / j_{c}(0)$ and $t=T / T_{c}$. We analyzed the $j_{c}(t)$ dependencies of superconducting strips prepared from granular YBCO films (solid and open circles in dependencies $a, b$ ) in terms of weak link connections between grains, where the power dependence $j_{c}(t)=j_{c}(0)(1-t)^{\alpha}$ occurs and $\alpha$ characterizes the type of current transport through weak links. The fitted dependencies with $\alpha \geq 2.5$ (dashed line, curve a) are characteristic for YBCO films with depressed $T_{c}$ and low critical current density $\left[j_{c}(0)<10^{3} \mathrm{~A} / \mathrm{cm}^{2}\right.$ at selffield conditions] and can be ascribed to nonhomogeneous superconductors with large dispersion of individual connections in weak link network of our granular films [2,3]. In case of YBCO films, where $\alpha \approx 1.5-2$ (dashed line $b$ in Fig. 1), weak links of $S c S$ (superconductor-constriction-superconductor) or SNS (superconductor-normal metal-superconductor) type dominate. The maximal critical current density of these samples was $j_{c}(0) \approx$ $\approx 10^{4} \mathrm{~A} / \mathrm{cm}^{2}$. On the basis of experimental observations one can conclude that the $\mathrm{dc}$ transport properties of granular films are determined by weak links between the superconducting grains, thus strongly reducing much higher critical current density of film grains [2].

The temperature dependence of critical current density of stripes, patterned from preferentially oriented YBCO thin films, exhibits downward curvature of $j_{c}(t)$ (solid and open triangles on curve $c$ ). In this case a new mechanism of critical current limitation begins to dominate. We ascribe this 


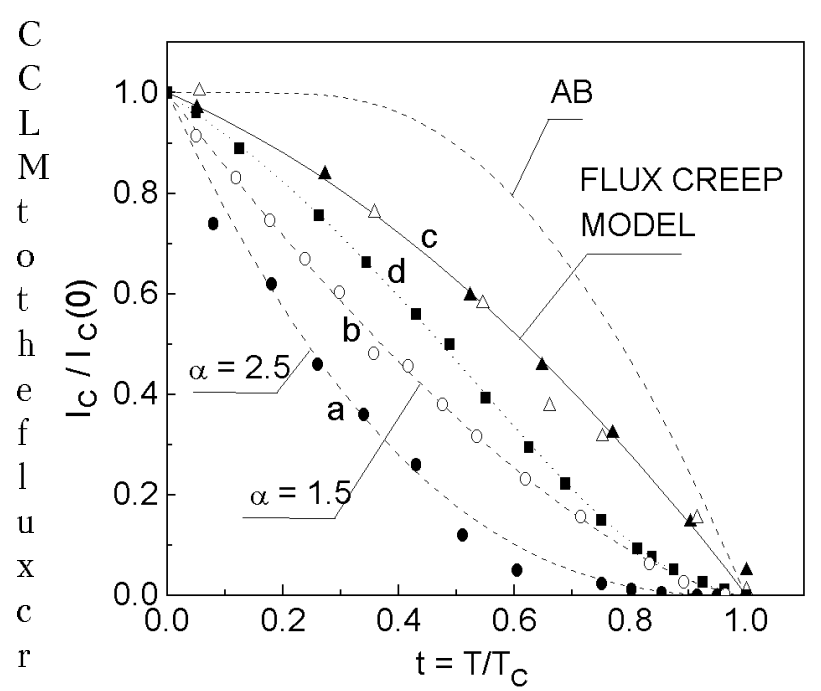

$\mathrm{e}_{\text {Fig. }}$ 1. Temperature dependence of the critical current density $\mathrm{e}_{\mathrm{of}}$ YBCO thin film strips of different quality (see text).

$\mathrm{p}$

and the full line (curve $c$ ) represents the fit of experimental data computed from relation $[4,5]$

$$
j_{c}(t)=j_{c}(0)\left(1-m t-n t^{2}\right)
$$

where $m=0.5, n=0.53$ and $j_{c}(0)=2.6 \cdot 10^{6} \mathrm{~A} / \mathrm{cm}^{2}$. Because this type of $j_{c}(t)$ is characteristic for samples with $j_{c}(0) \approx 10^{6} \mathrm{~A} / \mathrm{cm}^{2}$ we suppose that the intergranular weak links are not responsible for critical current limitation. The current-voltage $(I-V)$ characteristics of these samples in the low voltage range (just above $I_{c}$ ) also confirmed the flux-creep dissipation process [5].

The strips patterned from YBCO thin films epitaxially grown on $\mathrm{SrTiO}_{3}$ single crystal substrate $\left(j_{c}(0) \geq 10^{7} \mathrm{~A} / \mathrm{cm}^{2}\right)$ usually displayed the $j_{c}(t)$ dependence corresponding to curve $d$ in Fig. 1. The study of these temperature dependencies of critical current indicated a Ginzburg-Landau (GL) depairing mechanism in the temperature range close to $T_{c}$ [6]. The critical current of the microstrip approaches GL depairing current $I_{d}(t)[1,7]$ when the width $w$ and thickness $d$ of the strip are smaller or comparable to the transverse magnetic penetration depth of the thin film

$$
\lambda_{\perp}=\lambda \operatorname{coth}(d / 2 \lambda)
$$

where $\lambda$ is London penetration depth. If we consider the temperature dependence of magnetic field penetration depth $\lambda(t)=\lambda(0) /\left(1-t^{4}\right)^{1 / 2}$ for temperature close to $T_{c}$ we obtain $\lambda_{\perp} \approx 5-10 \mu \mathrm{m}$ which fulfill the conditions $w \leq \lambda_{\perp}$ and $d<<\lambda_{\perp}[\lambda(0)=200 \mathrm{~nm}$ and $d=50 \mathrm{~nm}$ were used in Eq. (2)], i.e. homogeneous cross-sectional distribution of transport current in the strip. In Fig. 2 the experimental $j_{c}(t)$

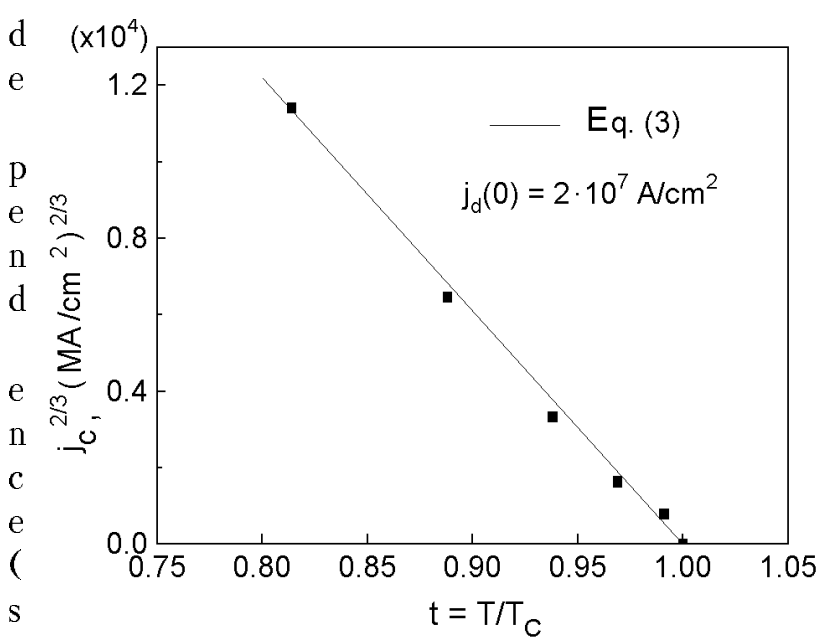

Fig. 2. Ginzburg-Landau depairing critical current density limiltation (full line) for «narrow» YBCO strips close to the critiical temperature.

d

squares) is compared with GL depairing current density (full line)

$$
j_{d}(t)=j_{d}(0)(1-t)^{3 / 2}
$$

where $j_{d}(0)=\left[\Phi_{0} / 3 \sqrt{3} \mu_{0} \pi \lambda^{2}(0) \xi(0)\right]$ is the depairing current density at $T=0 ; \Phi_{0}$ is magnetic flux quantum and $\xi(0)$ is coherence length. The value $j_{d}(0) \approx$ $\approx 2 \cdot 10^{7} \mathrm{~A} / \mathrm{cm}^{2}$ extrapolated from experimental dependence is in reasonable agreement with the value computed from (Eq. (3)): $j_{d}(0) \approx 8 \cdot 10^{7} \mathrm{~A} / \mathrm{cm}^{2}$ for $\lambda(0) \approx 200 \mathrm{~nm}$ and $\xi(0) \approx 3 \mathrm{~nm}$. These experimental results have shown that $j_{c}(t)$ close to $T_{c}$ may approach the GL depairing current as a limiting mechanism.

In the low temperature range (the dependence $d$ in Fig. $1, t<0.6$ ) where $w>\lambda_{\perp}$ (wide strip) another CCLM takes place. Due to current concentration at the strip edges, the edge barrier to vortex entry is lowered, and dissipation process in microstrip starts at the current value [8]

$$
I_{c 1} \approx\left(\Phi_{0} / \mu_{0} \lambda_{\perp}\right) \ln (d / 4 \xi)
$$

the «lower» critical current. The depression of $j_{c}(t)$ more than one order of magnitude [for wide strips $w>\lambda_{\perp}(t)$ ], in comparison to $j_{d}(t)$, can be explained by the entering of Abrikosov vortices into the superconductor and their motion across the strip [6].

Phase-slip phenomena (PSP) in the one-dimensional superconducting strips [9] as well as in wide strips (moving phase-slip centers or phase-slip lines), may occur as CCLM [1]. In this case the temperature dependence of the critical current density is described by the relation $j_{c}(t)=j_{c}(0)(1-t)^{3 / 2}$, predicted by GL theory [Eq. (3), but $j_{c}(0)<j_{d}(0)$ ], and the dissipation process is characterized by 


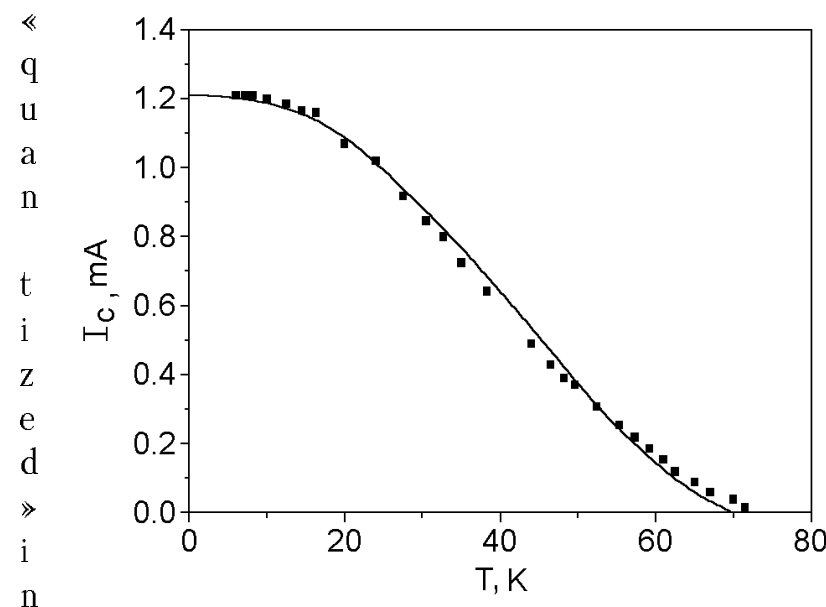

Fig. 3. The dependence of critical current $I_{c}$ vs. temperature of a bicrystal grain boundary Josephson junction. Full line: modified Usadel equation for $L / \xi_{N}=0.6-4.2$.

$\mathrm{e}$

ases of differential resistance in the $I-V$ characteristic. We observed properties similar to PSP in $10-\mu \mathrm{m}$ wide and $100 \mu \mathrm{m}$ long granular YBCO strips [10]. The estimated quasiparticle diffusion length $(\approx 25 \mu \mathrm{m})$ and temperature dependence of current at voltage steps (i.e. differential resistance increases) are in accordance with the PSP theory. We have concluded that grain boundaries and quality of the strip edges play very important roles in the PSP, and in the critical current limitation in wide as well as narrow YBCO strips.

We also investigated the $I_{c}(T)$ dependencies of YBCO grain boundary Josephson junctions of bicrystal type [11]. The most adequate fit (full line in Fig. 3) to the experimental $I_{c}(T)$ dependence (solid squares) we explained by the model of the temperature dependent ratio $L / \xi_{N}$, where $L$ is the length of a normal region in the YBCO strip at a bicrystal boundary in the direction of current flow and $\xi_{N}$ is the coherence length of the normal region. In this case the temperature dependent transparency of the junction normal region for superconducting carriers is the main factor of CCLM. The Ambegaokar-Baratoff [12] $I_{c}(t)$ dependence (AB in Fig. 1) in our studies is not suitable for approximation of any one dependence for YBCO strips, sandwich YBCO/PrBaCuO/YBCO [13] or bicrystal grain boundary $S N S$ junctions.

\section{Summary}

In this contribution we reviewed various mechanisms of critical current limitation in high $T_{c}$ superconducting YBCO thin film strips and structures. We analyzed these mechanisms on the base of the temperature dependence of the critical current and corresponding models that are valid for low $T_{c}$ superconductors. The weak links, mainly of $S N S$ and $S c S$ types, flux-creep, Ginzburg-Landau depairing current, phase slip phenomena and temperature dependent «transparency» of the bicrystal junction for transport superconducting current are responsible for critical current limitation in high temperature superconducting thin film samples.

The autors are obliged to Prof. I. M. Dmitrenko for great many fruitful working discussions and human contacts in Bratislava and Kharkov, and they wish to dear I. M. even better years of productive work, health and further close contacts with group in Bratislava.

1. I. M. Dmitrenko, Low. Temp. Phys. 22, 648 (1996).

2. V. Strbik, S. Benacka, S. Gazi, S. Chromik, J. Levarsky, and J. Sith, Mod. Phys. Lett. B3, 729 (1989).

3. N. Savvides, Physica C165, 371 (1990).

4. J. Mannhart, P. Chaudhari, O. Dimos, C. C. Tsuei, and T.R. McGuire, Phys. Rev. Lett. 61, 2476 (1988).

5. V. Strbik, R. Adam, S. Benacka, and S. Chromik, Proc. of the Intern. Workshop on Critical Current Limitations in High Temperature Superconductors Zaborow (Poland) (1991) p. 366.

6. V. Strbik, S. Benacka, S. Chromik, R. Adam, P. Tomas, E. Pincik, and V. Smatko, presented on Inernational Conference: Critical Currents in High $T_{c}$ Superconductors, Vienna (1992).

7. K. K. Likharev, Zh. Eksp. Teor. Fiz. 61, 1700 (1971) [Sov. Phys. JETP 34, 906 (1972)].

8. K. K. Likharev, Radiofizika 14, 919 (1971).

9. W. J. Skocpol, M. R. Beasley and M. Tinkham, J. Low Temp. Phys. 16, 145 (1974).

10. V. Strbik, R. Adam, and S. Benacka, Proc. of Weak Superconductivity Symposium, Smolenice (Slovak Republic) (1989) p. 75

11. V. Strbik, O. Harnack, S. Chromik, M. Darula, and S. Benacka, Applied Superconductivity 1997, Proc. of EUCAS'97, H. Rogalla and D. H. A. Blank (eds), Inst. of Physics, Conf. series No. 158, p. 503.

12. V. Ambegaokar and A. Baratoff, Phys. Rev. Lett. 10, 486 (1963).

13. Unpublished results of authors. 